\title{
Macrophages and neutrophils are the targets for immune suppression by glucocorticoids in contact allergy
}

\author{
Jan P. Tuckermann, ${ }^{1,2}$ Anna Kleiman, ${ }^{1,2}$ Richard Moriggl, ${ }^{3}$ Rainer Spanbroek, ${ }^{4}$ Anita Neumann, ${ }^{2}$ \\ Anett Illing, ${ }^{2}$ Björn E. Clausen, ${ }^{5}$ Brenda Stride, ${ }^{1,6}$ Irmgard Förster, ${ }^{7}$ Andreas J.R. Habenicht, ${ }^{4}$ \\ Holger M. Reichardt,, ${ }^{8}$ François Tronche, ${ }^{9}$ Wolfgang Schmid, ${ }^{1}$ and Günther Schütz ${ }^{1}$
}

\begin{abstract}
1Division of Molecular Biology of the Cell I, German Cancer Research Center, Heidelberg, Germany. ${ }^{2}$ Division of Tissue-Specific Hormone Action, Leibniz Institute for Age Research, Fritz Lipmann Institute, Jena, Germany. ${ }^{3}$ Ludwig Boltzmann Institute for Cancer Research, Vienna, Austria.

${ }^{4}$ Institute for Vascular Medicine, Friedrich Schiller University, Jena, Germany. ${ }^{5}$ Department of Cell Biology and Histology, Academic Medical Center, University of Amsterdam, Amsterdam, The Netherlands. ${ }^{6}$ European Molecular Biology Laboratory (EMBL), Heidelberg, Heidelberg, Germany. ${ }^{7}$ Institut für Umweltmedizinische Forschung, Heinrich-Heine-Universität Düsseldorf, Düsseldorf, Germany. ${ }^{8}$ Department of Cellular and Molecular Immunology, University of Göttingen, Göttingen, Germany. "“Génétique moléculaire, neurophysiologie et comportement”, Collège de France, UMR7148 CNRS, Paris, France.
\end{abstract}

\begin{abstract}
Glucocorticoids (GCs) are widely used in the treatment of allergic skin conditions despite having numerous side effects. Here we use Cre/loxP-engineered tissue- and cell-specific and function-selective GC receptor (GR) mutant mice to identify responsive cell types and molecular mechanisms underlying the antiinflammatory activity of GCs in contact hypersensitivity (CHS). CHS was repressed by GCs only at the challenge phase, i.e., during reexposure to the hapten. Inactivation of the GR gene in keratinocytes or $T$ cells of mutant mice did not attenuate the effects of GCs, but its ablation in macrophages and neutrophils abolished downregulation of the inflammatory response. Moreover, mice expressing a DNA binding-defective GR were also resistant to GC treatment. The persistent infiltration of macrophages and neutrophils in these mice is explained by an impaired repression of inflammatory cytokines and chemokines such as IL-1 $\beta$, monocyte chemoattractant protein- 1 , macrophage inflammatory protein- 2 , and IFN- $\gamma$-inducible protein 10. In contrast TNF- $\alpha$ repression remained intact. Consequently, injection of recombinant proteins of these cytokines and chemokines partially reversed suppression of CHS by GCs. These studies provide evidence that in contact allergy, therapeutic action of corticosteroids is in macrophages and neutrophils and that dimerization $G R$ is required.
\end{abstract}

\section{Introduction}

Contact dermatitis is a common condition caused by exposure to low molecular weight compounds such as metals or poison ivy. It is usually treated by topical or systemic application of glucocorticoids (GCs) (1). Such treatment effectively ameliorates the ongoing inflammatory response, but the long-term use of corticosteroids can cause adverse effects including skin atrophy or osteoporosis (2). While the general antiinflammatory capacity of GCs is well documented, their target cells and the transcriptional mechanisms underlying the treatment of allergic conditions by GCs remain largely elusive.

Contact hypersensitivity (CHS), a T cell-dependent immune response, is a classic mouse model of human allergic contact dermatitis. It involves 2 phases known as the sensitization phase and the challenge phase (3). In the sensitization phase, the hapten is cross-linked to epidermal proteins and taken up by resident epidermal Langerhans cells (LCs), dermal DCs, and tissue macrophages. Subsequently, these cells - triggered by cytokines such as TNF- $\alpha$ and IL- $1 \beta$ - migrate to the draining lymph nodes

Nonstandard abbreviations used: BMDM, BM-derived macrophages; CHS, contact hypersensitivity; DNFB, 2,4-dinitrofluorobenzene; EGFP, enhanced GFP; GC, glucocorticoid; GR, GC receptor; GRE, GC-responsive element; IP-10, IFN- $\gamma$-inducible protein 10; LC, Langerhans cell; MCP-1, monocyte chemoattractant protein-1; MIP-2, macrophage inflammatory protein-2.

Conflict of interest: The authors have declared that no conflict of interest exists. Citation for this article: J. Clin. Invest. 117:1381-1390 (2007). doi:10.1172/JCI28034. and mature into potent antigen presenting cells (APCs) (4). In turn, naive $T$ cells are activated and differentiate into antigenspecific Th1 and cytotoxic T cells under the influence of polarizing signals such as IL-12 (5). These T cells are then designated as "sensitized." In parallel, B-1 cells in the peritoneal cavity are stimulated by IL-4-secreting NKT cells to produce antigen-specific IgM. In addition, NK cells appear to play a role in sensitization (6-8). In the challenge phase, initiated by the reexposure to the same hapten, cross-linking of IgM molecules causes the release of the complement factor C5a (9). This acts on platelets, skin resident mast cells, and macrophages (10) and thereby results in the activation of the microvasculature (11), allowing for the penetration of a first wave of leukocytes within 2 hours (12). In response to chemokines such as IFN- $\gamma$-inducible protein 10 (IP-10, also known as CXCL10), sensitized T cells enter the dermis at the site of hapten exposure and become restimulated by resident APCs (13). These T cells then release proinflammatory mediators, which trigger resident myeloid cells to secrete chemokines such as macrophage inflammatory protein-2 (MIP-2, also known as CXCL2) and monocyte chemoattractant protein-1 (MCP-1, also known as CCL2), leading to a massive second wave of leukocyte infiltration (4).

GCs are known to suppress a plethora of proinflammatory genes encoding cytokines, chemokines, cell adhesion molecules, and other mediators, thereby interfering with the inflammatory response (14). These effects are mediated by the GC receptor (GR), 
A

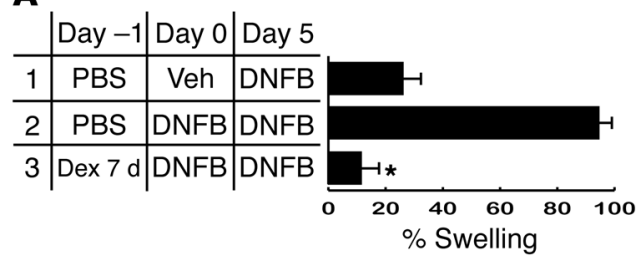

C

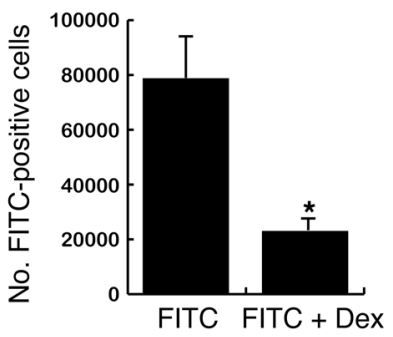

B

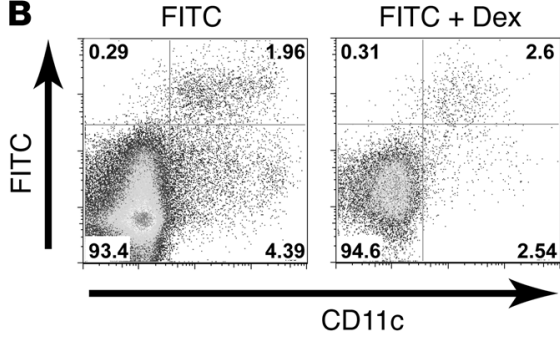

D

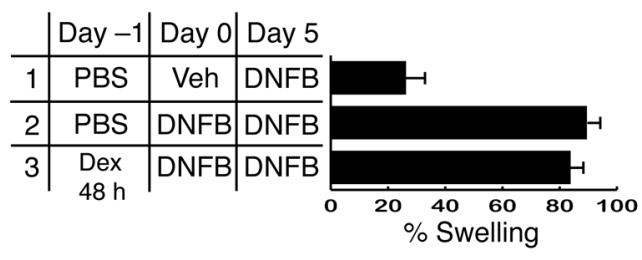

Figure 1

Dexamethasone treatment suppresses CHS but is ineffective when applied during the sensitization phase. (A) Beginning at day -1 and continuing for 7 days, mice were treated with either PBS (rows 1 and 2 ) or dexamethasone (Dex; row 3). On day 0, mice were treated on the shaved back skin either with vehicle (row 1) or with DNFB (rows 2 and 3). All mice were challenged with DNFB at the ear on day 5 , the effect of which was determined 24 hours later. ${ }^{*} P<0.01(n=6)$. (B and $\left.\mathbf{C}\right)$ Wild-type mice were injected i.p. with either dexamethasone or vehicle. Twenty-four hours later, they were topically treated on the shaved back skin with the fluorophore FITC. After 18 hours, cells from regional lymph nodes were analyzed by flow cytometry. (B) FACS analysis of lymph node cells was performed to determine the level of expression of CD11c and loading with FITC. (C) Numbers of FITC-positive cells after treatment with either FITC alone or FITC in combination with dexamethasone. ${ }^{*} P<0.01$. (D) Mice were treated as described in $\mathbf{A}$ except that the dexamethasone treatment was terminated at day 1 after DNFB sensitization (Dex 48 h; row 3$)(n=6)$.

a transcription factor belonging to the nuclear receptor superfamily. The GR controls transcription by different mechanisms (15). One mechanism requires binding of receptor homodimers to GC-responsive elements (GREs) in gene regulatory regions. In a second mechanism the GR also has the ability to modulate the activity of other transcription factors such as AP- $1, \mathrm{NF}-\kappa \mathrm{B}$, and STAT5 independent of dimerization and subsequent DNA binding of the receptor (reviewed in refs. 15, 16). We previously demonstrated that AP-1- and NF- $\mathrm{BB}-$ dependent proinflammatory genes were effectively repressed in mice carrying a point mutation that impairs dimerization-induced DNA binding of the $\operatorname{GR}\left(\mathrm{GR}^{\mathrm{dim}}\right)(17,18)$. In addition, phorbol ester-induced irritative inflammation was effectively suppressed by GCs in these animals (17). Here we asked whether intervention with GCs of a $\mathrm{T}$ cell-dependent delayed-type hypersensitivity reaction requires DNA binding of the GR.

To identify the phase, cell types, and mechanisms of GR action that are involved in the repression of an allergic skin condition by GCs, we induced CHS in various GR-mutant mouse strains. Our results demonstrate that only the challenge phase is responsive to GCs, that myeloid cells are the primary targets of these actions, and that these effects require DNA binding by the GR. The analysis of macrophages in cell culture further indicates that downregulation of cytokines such as IL-1 $\beta$ and chemokines such as MCP-1, MIP-2, and IP-10 is impaired. Application of these proteins in part counteracts the antiinflammatory effects of GCs in CHS. Thus, our results shed new light on the mechanisms of these drugs in the treatment of allergic skin conditions. applied FITC as a contact sensitizer. Dexamethasone treatment strongly reduced the number of FITC-loaded CD $11 \mathrm{c}^{+}$cells found in the draining lymph nodes after 19 hours (Figure 1, B and C). However, the numbers of $\mathrm{T}$ and $\mathrm{B}$ cells were also reduced (data not shown). These data are comparable with earlier studies that describe a rapid effect of GCs on DC migration (19).

We then tested whether the reduction of hapten-loaded DCs in lymph nodes during the sensitization phase had any effect on the inflammatory response (Figure 1D). To this end, dexamethasone treatment was started 1 day before the first application of DNFB and lasted 48 hours until day 1 as specified in Methods. On day 5 after sensitization, the mice were re-challenged with DNFB, and 24 hours later the swelling was determined. Interestingly, systemic treatment with dexamethasone during the sensitization phase did not prevent the inflammatory response evoked by the second application of DNFB (Figure 1D). Similarly, topical treatment during the sensitization phase with fluocinonide-containing ointment, a commercially available synthetic GC preparation, was unable to inhibit the swelling response (data not shown). Thus, the diminished DC and $\mathrm{T}$ cell numbers in the draining lymph nodes after dexamethasone treatment suffice to allow effective $\mathrm{T}$ cell priming by the hapten.

Since GCs apparently do not alter the sensitization phase of CHS, we assumed that the challenge phase was responsive to the treatment. Therefore, we applied dexamethasone only at day 5 , 1 hour prior to the second DNFB application (Figure 2A). GC application at this time point effectively prevented the inflammatory response. A similar inhibition, in concordance with the 
A

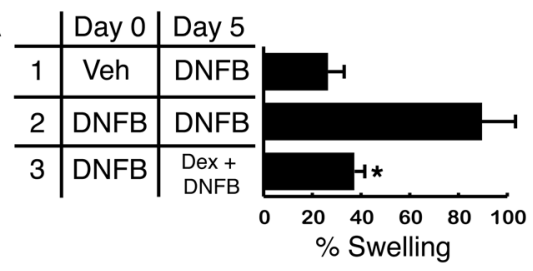

B

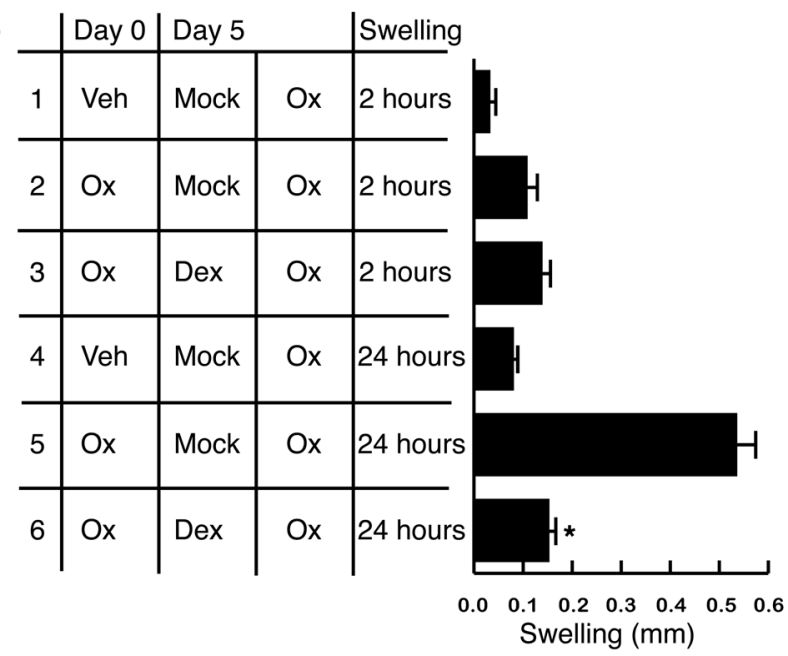

clinical practice, was also achieved by topical application of fluocinonide ointment (data not shown).

The effector phase of CHS consists of 2 waves of leukocyte infiltration (20). An early wave occurs 2 hours after application of the contact sensitizer and is elicited by $\operatorname{IgM}$ and C5a, which activate mast cells. Those in turn act on vascular endothelial cells, leading to the migration of sensitized $\mathrm{T}$ cells to the site of hapten application $(9,12)$. This early invasion is essential for a second wave of leukocyte infiltration, which leads to the development of the full inflammatory response (12). Since the initial swelling is comparably weak, we used the strong contact sensitizer oxazolone to dissect the effects of dexamethasone on the 2 phases. GC treatment of oxazolone-sensitized mice 1 hour before the rechallenge with oxazolone did not substantially affect the early response measured after 2 hours (Figure 2B, compare rows 2 and 3 ). In contrast, the late phase of the CHS response measured after 24 hours was strongly repressed by this treatment (Figure 2B, compare rows 5 and 6). In conclusion, GCs repress $\mathrm{CHS}$ during the late challenge phase without interfering with the early wave of leukocyte infiltration.

Immunosuppressive functions of GCs require the presence of the GR in myeloid cells. Next, we wished to identify the cell types sensitive to GC action by the use of conditional GR mutant mice. We crossed K14CreERT2 transgenic mice (21) with GR flox mice (22) to study whether effects on keratinocytes contribute to the observed suppression of CHS. After we induced recombination of the $\mathrm{GR}^{\text {flox }}$ allele with tamoxifen, these $\mathrm{GR}^{\mathrm{K} 14 \mathrm{Cre}}$ mice selectively lacked the receptor in keratinocytes (data not shown). Administration of DNFB induced a massive swelling response, which was effectively repressed by dexamethasone irrespective of the genotype (Figure 3A). Thus the presence of the GR in keratinocytes is not essential for the antiinflammatory effect of dexamethasone in the treatment of CHS.

\section{Figure 2}

Dexamethasone inhibits edema formation during the challenge phase of $\mathrm{CHS}$. (A) Mice were treated on day 0 as described in Figure $1 \mathrm{~A}$ with either vehicle alone (row 1) or DNFB on the shaved back skin (rows 2 and 3). All mice were treated with DNFB on the ear on day 5 to evoke a challenge response. Mice were additionally treated with PBS (rows 1 and 2) or dexamethasone (row 3) starting 1 hour before the reapplication of DNFB at day 5 and dexamethasone was supplied in the drinking water until day $6 .{ }^{*} P<0.01(n=5)$. (B) Animals were treated with vehicle (rows 1 and 4 ) or oxazolone (Ox) on the back skin on day 0 (rows $2,3,5$, and 6 ). On day 5 , mice were mock treated with vehicle (rows 1, 2, 4, and 5) or with dexamethasone (rows 3 and 6). One hour later, all mice were challenged on the ear with oxazolone. The ear swelling was determined 2 hours (rows 1-3) or 24 hours (rows 4-6) after challenge. ${ }^{*} P<0.01(n=6)$.

Because CHS is a T cell-dependent hypersensitivity response, we next analyzed the capacity of GCs to repress inflammation in mice carrying a T cell-specific deletion of the GR gene (GR ${ }^{\text {lckCre }}$ mice). These animals were generated by crossing $\mathrm{GR}^{\text {flox }}$ mice (22) with lckCre transgenic mice (23), which display an effective and selective disruption of the GR gene only in the T cell lineage (24). Unexpectedly, the dexamethasone-mediated suppression of CHS, induced by either DNFB or oxazolone, was completely unaffected in $\mathrm{GR}^{\text {lckCre }}$ mice (Figure $3 \mathrm{~B}$ and data not shown). Consequently, the control of $\mathrm{T}$ cell functions by GCs is also dispensable for the inhibitory action of dexamethasone on CHS.

Since the second wave of leukocyte infiltration is primarily mediated by neutrophils and macrophages, we studied mice that lack the GR in myeloid cells (GR LysMCre). The animals were generated by crossing $\mathrm{GR}^{\text {flox }}$ mice with lysozyme $\mathrm{M}$ Cre knockin mice (25). To determine the recombination efficiency in various myeloid cell types, GR ${ }^{\text {LysMCre }}$ mice were mated with tk-loxPenhanced GFP (tk-loxP-EGFP) reporter (RA/EG) animals (26). Cells from bone marrow, spleen, and lymph nodes were isolated and analyzed by flow cytometry (Figure 3C). Almost complete recombination of the loxP sites was achieved in neutrophils (more than $90 \%$ ) and macrophages (70\%), whereas recombination in LCs (43\%), DCs (16\%), and mast cells (26\%) was less effective (Table 1). T and B cells were largely unaffected in these mice (data not shown). Effective disruption of the GR gene in neutrophils was further demonstrated by the complete conversion of the $\mathrm{GR}^{\text {flox }}$ allele into the $\mathrm{GR}^{\text {null }}$ allele in FACS-sorted $\mathrm{EGFP}^{+} \mathrm{GR}-1^{+}$ granulocytes (Figure 3D). Whereas DNFB-induced swelling was normally repressed by dexamethasone in control mice, the same treatment completely failed to suppress the inflammatory response in $\mathrm{GR}^{\text {LysMCre }}$ mice (Figure $3 \mathrm{E}$ ). Histological analysis demonstrated the persistence of massive leukocyte infiltration in the mutant mice despite treatment with GCs (data not shown). These results demonstrate that GR-controlled functions in macrophages and neutrophil granulocytes are essential for the suppression of CHS by GCs, indicating that these cells are the primary targets of dexamethasone-mediated therapy.

DNA binding of the GR is required for the suppression of CHS by GCs. To define the mechanism of GR action in the suppression of CHS, we took advantage of the function-selective GR dim mutation. We have previously demonstrated that interference with dimerization-induced DNA binding in GR ${ }^{\mathrm{dim}}$ mice does not impair the antiinflammatory activity of GCs in irritative inflammation (17). To determine whether GCs are similarly able to suppress a T cell-dependent allergic inflammation in GR ${ }^{\mathrm{dim}}$ mice, we induced 

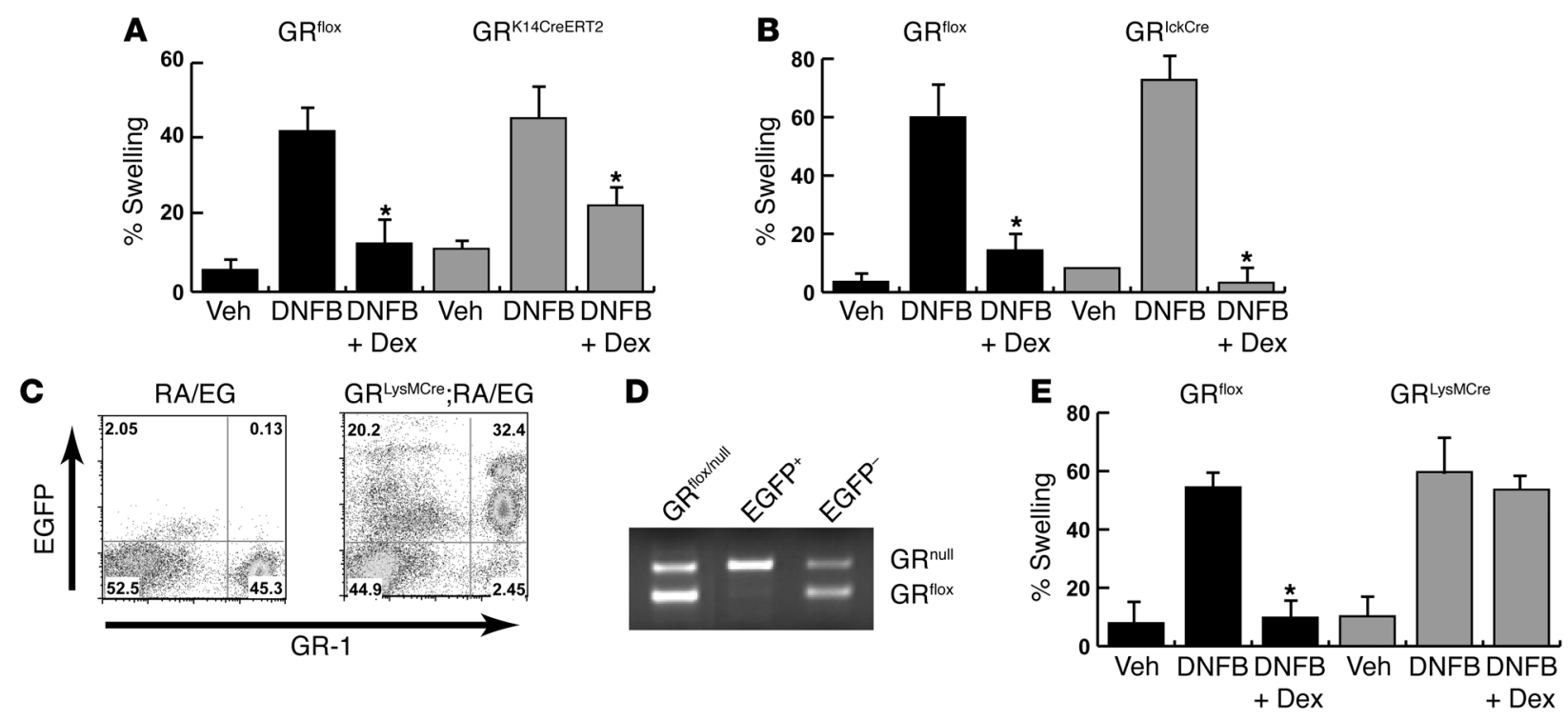

Figure 3

GC-mediated suppression of CHS requires GR actions in myeloid cells. (A) Swelling response of GR ${ }^{\text {flox }}$ and GR G $^{\mathrm{K} 14 \mathrm{CreERT2}}$ mice (shown as \% increase in thickness; $\left.{ }^{*} P<0.01 ; n=5-6\right)$. All mice were sensitized at day 0 with vehicle (Veh) or DNFB and challenged with DNFB in the presence or absence of dexamethasone. (B) Swelling response of $\mathrm{GR}^{\text {flox }}$ and $G R^{\text {lckCre }}$ mice treated as described in A. (C) FACS analysis of BM cells from GRLysMCre mice crossed with RA/EG mice containing a tk-loxP-EGFP reporter gene (GRLysMCre;RA/EG mice) to determine the expression of EGFP and GR-1. Numbers indicate the percentage of cells in each quadrant. (D) PCR analysis of genomic DNA isolated from GR-1+ granulocytes positive or negative for EGFP from GR ${ }^{\text {LysMCre }}$;RA/EG mice in comparison with genomic DNA derived from $\mathrm{GR}^{\text {flox }} / \mathrm{GR}^{\text {null }}$ heterozygous mice (50\% per allele). (E) Swelling response of $\mathrm{GR}^{\text {flox }}$ and $\mathrm{GR}^{\mathrm{LysMCre}}$ mice treated as described in $\mathbf{A}$.

CHS with DNFB (Figure 4A) and oxazolone (Figure 4B), followed by administration of dexamethasone during the challenge phase. Unexpectedly, the inflammatory response was hardly affected by dexamethasone treatment in $\mathrm{GR}^{\mathrm{dim}}$ mice in contrast to wild-type mice (Figure 4, A and B). Histological examination of inflamed ears after oxazolone treatment revealed a strong infiltration by leukocytes in wild-type and $\mathrm{GR}^{\mathrm{dim}}$ mice. As expected, ears of wildtype mice showed only few infiltrating cells when treated with oxazolone and dexamethasone. However, in GR ${ }^{\text {dim }}$ ears dexamethasone did not prevent a persistent accumulation of leukocytes (Figures 5 and 6). The number of $\mathrm{CD}^{+} 8^{+}$macrophages located in the dermis was not diminished in ear skin of GR ${ }^{\text {dim }}$ mice (Figure 5). Similarly, massive infiltration by GR-1 $1^{+}$neutrophils of dermis and epidermis after oxazolone treatment was reduced by dexamethasone only in the skin of wild-type but not GR ${ }^{\mathrm{dim}}$ mice (Figure 6). These results indicate that dimerization of the GR and GRE-dependent transcription (27) are essential for the suppression of an allergic inflammation.

Similar impairment of GC suppression of leukocyte infiltration in the CHS response in GR LysMCre mice and GR ${ }^{\text {dim }}$ mice indicates that factors that fail to be regulated in myeloid cells from $\mathrm{GR}^{\mathrm{dim}}$ mice may represent essential targets of GC action in CHS. Therefore, we determined the level of release of proinflammatory cytokines and chemokines by BM-derived macrophages (BMDMs) of wild-type and GR dim mice. TNF- $\alpha$, IL-1 $\beta$, MCP-1, MIP-2, and IP-10 are important mediators of leukocyte migration in CHS (4). Dexamethasone repressed LPS-induced TNF- $\alpha$ release to a similar extent in wild-type and $\mathrm{GR}^{\mathrm{dim}}$ cells (Figure 7A). In contrast, inhibition of IL-1 $\beta$ and MCP- 1 release by GCs was significantly impaired in $\mathrm{GR}^{\mathrm{dim}}$ cells (Figure 7, B and C). Importantly, the synthesis of the MIP-2 and IP-10 was hardly repressed by dexamethasone in GR ${ }^{\operatorname{dim}}$ macrophages (Figure 7, D and E). It is noteworthy that protein levels were regulated to a similar extent as the mRNA levels of the respective genes (Supplemental Figure 1; supplemental material available online with this article; doi:10.1172/JCI28034DS1). These data suggest that impaired downregulation of IL-1 $\beta$, MCP-1, MIP-2, and IP-10 may contribute to the persistent inflammation during CHS in dexamethasone-treated $\mathrm{GR}^{\mathrm{dim}}$ mice.

The functional relevance of the factors analyzed for the repression of CHS by GCs was confirmed by local administration of recombinant proteins to dexamethasone-treated mice during the challenge phase (Figure 8). The antiinflammatory effect of dexamethasone was significantly impaired by exogenous IL- $1 \beta$, MCP-1, MIP-2, and IP-10 $(P<0.05$; Figure 8 , A-D) but not by application of TNF- $\alpha(P=$ NS; Figure $8 \mathrm{E})$. Administration of cytokines or chemokines tested individually did not evoke a complete reversal of GC-induced repression of CHS, which suggests that they might act in synergy or that other proteins might contribute. However, our findings do indicate that these proinflammatory mediators participate in the suppressive activity of GCs in the treatment of allergic skin conditions.

\section{Discussion}

GCs applied at pharmacological doses are strong inhibitors of contact dermatitis, which makes them the treatment of choice for this condition. Here we describe molecular and cell type-specific mechanisms underlying the beneficial effects of GCs, exemplified by CHS, a classic rodent model of human allergic dermatitis. We show that the immune response elicited by reexposure to hapten is not suppressed by GCs during the sensitization phase but is strongly reduced when applied during the challenge 
Table 1

Recombination efficiency of a tk-flox-EGFP allele in LysM-Cre knockin mice

Cell type
Neutrophils
Macrophages
DC (lymph node)
DC (spleen)
LC (skin)
DC (BM)
BMMC

Values were determined as described in Figure $3 \mathrm{C}$ and summarize the results obtained for various cell types and the surface markers used for their characterization. BMMC, BM-derived mast cell.

phase. In this phase the presence of the GR in keratinocytes and $\mathrm{T}$ cells is dispensable, whereas GR expression is required in myeloid cells for effective immunosuppression. These GC actions depend on DNA binding of the GR and involve repression of IL-1 $\beta$, MCP-1, MIP-2, and IP-10.

In agreement with an earlier study (19), we observed that GCs applied during the sensitization phase of CHS suppressed the accumulation of APCs in the draining lymph nodes but not the development of an inflammatory response. Thus, GCs apparently do not interfere with $\mathrm{T}$ cell priming, which indicates that a reduced number of APCs in the lymph nodes still allows for the generation of sufficient antigen-specific $\mathrm{T}$ cells capable of initiating an immune response. This is in agreement with the previous observation that fewer than $50,000 \mathrm{DCs}$ are required to support CHS (28). In contrast, systemic as well as topical application of GCs during the challenge phase prevents an inflammatory response. However, this effect was limited to the late inflammatory reaction that consists of the influx of myeloid and $T$ cells after cytokine and chemokine release, whereas the early swelling response was unaffected.

Given the well documented role of T cells in CHS response, our observation that GCs strongly repress inflammation in mice lacking the GR in T cells was unexpected. Sensitized T cells were previously found to transfer antigen specificity to recipient animals (29). In addition, T cell-depleted, CD8-knockout, and CD4knockout mice exhibit an impaired CHS response $(5,30)$. While these experiments point to a central role of T cells in CHS, recent investigations showed that skin inflammation does not necessarily depend on T cells. In a psoriasis model established in keratinocyte-specific IKK2-knockout mice, the inflammatory response was retained after genetic ablation of T cells (31). In Rag2 $2^{--}$and SCID mice, hapten-specific CHS reactions occur normally as in wild-type mice (8). In our model, at least inhibition of $\mathrm{T}$ cell activity by GCs is not essential to block CHS. This is supported by the observation that GR ${ }^{\text {LysMCre }}$ mice, which carry a functional GR in $\mathrm{T}$ cells - but not in myeloid cells - are resistant to GC treatment. Keratinocytes participate in the pathogenesis of skin diseases (reviewed in ref. 32), e.g., by the release of TNF- $\alpha$ necessary for DC mobilization $(33,34)$. However, tamoxifen-treated GR ${ }^{\mathrm{K} 14 \mathrm{Cre}}$ mice, which lack the GR in the entire epidermis still exhibit a significant reduction of the swelling response after GC application. Keratinocytes are a source of CHS-mediating cytokines, but they are not a critical target for GCs in CHS. In contrast, we observed a complete block of GC actions on CHS in mice lacking the GR in myeloid cells ( $\left.\mathrm{GR}^{\mathrm{Lys} M \mathrm{Mre}}\right)$. These animals primarily lack the GR in macrophages and neutrophils, while DCs and mast cells are less affected by Cre-induced recombination. Although $42 \%$ of the LCs showed Cre-induced recombination, LCs should not be a major target for GCs because depletion of LCs by GCs or genetic ablation of LCs enhanced CHS responses $(35,36)$. Thus, the persistent activity of macrophages and neutrophils in the skin after GC treatment is the most likely reason for the impaired immunosuppression in $\mathrm{GR}^{\mathrm{Lys} M \mathrm{MCr}}$ mice. Interestingly, macrophages were recently shown to be required for neutrophil infiltration and inflammatory lesions in 2 models of psoriasis $(37,38)$. In one of them, namely mice with a hypomorphic CD18 allele, dexamethasone treatment was able to resolve this inflammation (39). These studies support our finding that GC action on macrophages is crucial for curing skin conditions.

Interestingly, repression of CHS responses via GCs in myeloid cells requires binding of the GR, as we demonstrate here. This is in contrast to our previous findings in an irritative inflammatory model of $\mathrm{GR}^{\mathrm{dim}}$ mice, in which inflammation was resolved by dexamethasone (17). In irritative skin inflammation, the lack of the GR in myeloid cells does not abrogate the antiinflammatory activity by GC. Thus, the requirement of GR DNA binding for the antiinflammatory activities of GCs appears to be important of certain immunological processes that involve myeloid cells as targets for GCs.

Infiltration of the skin by $\mathrm{CD} 68^{+}$macrophages and GR- $1^{+}$neutrophils in $\mathrm{GR}^{\mathrm{dim}}$ mice was not prevented by dexamethasone. This suggests that the persistent infiltration of leukocytes is likely due to inflammatory mediators that fail to be regulated by a dimerization- and DNA binding-deficient GR. The fact that both $\mathrm{GR}^{\mathrm{LysMCre}}$ and $\mathrm{GR}^{\mathrm{dim}}$ mice are resistant to GC-medi-
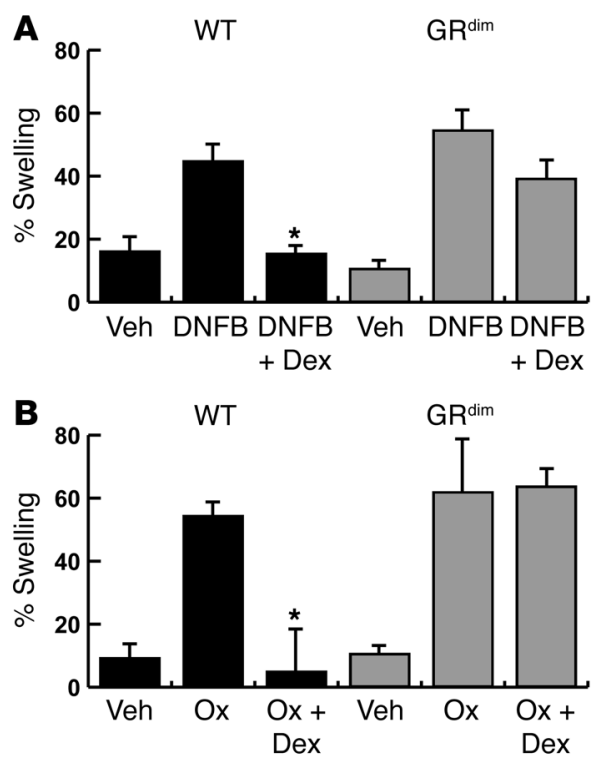

\section{Figure 4}

GC-mediated suppression of $\mathrm{CHS}$ is impaired in $\mathrm{GR}^{\text {dim }}$ mice. (A) Swelling response observed in wild-type and GR dim mice sensitized with vehicle or DNFB. Mice were challenged with DNFB in the presence or absence of dexamethasone. ${ }^{*} P<0.01(n=5-6)$. (B) Swelling response in wild-type and $\mathrm{GR}^{\text {dim }}$ mice sensitized with vehicle or oxazolone. Mice were challenged with oxazolone in the presence or absence of dexamethasone. ${ }^{*} P<0.01(n=5-6)$. 
WT
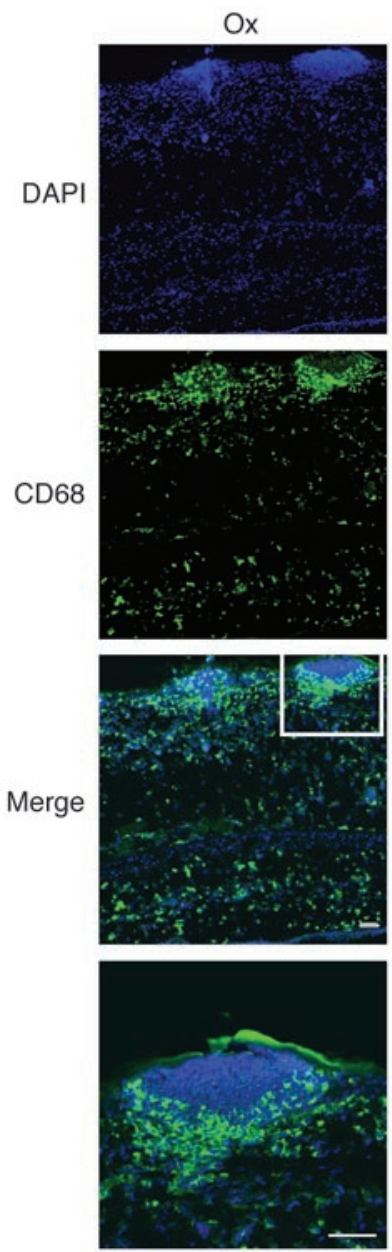
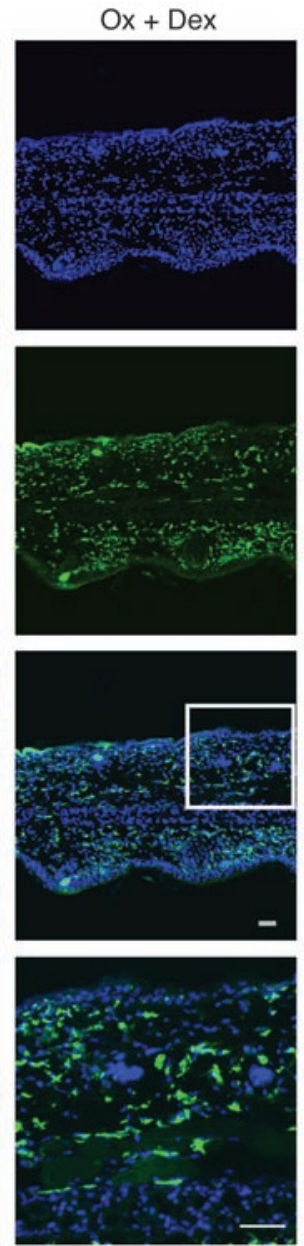

$\mathrm{GR}^{\mathrm{dim}}$
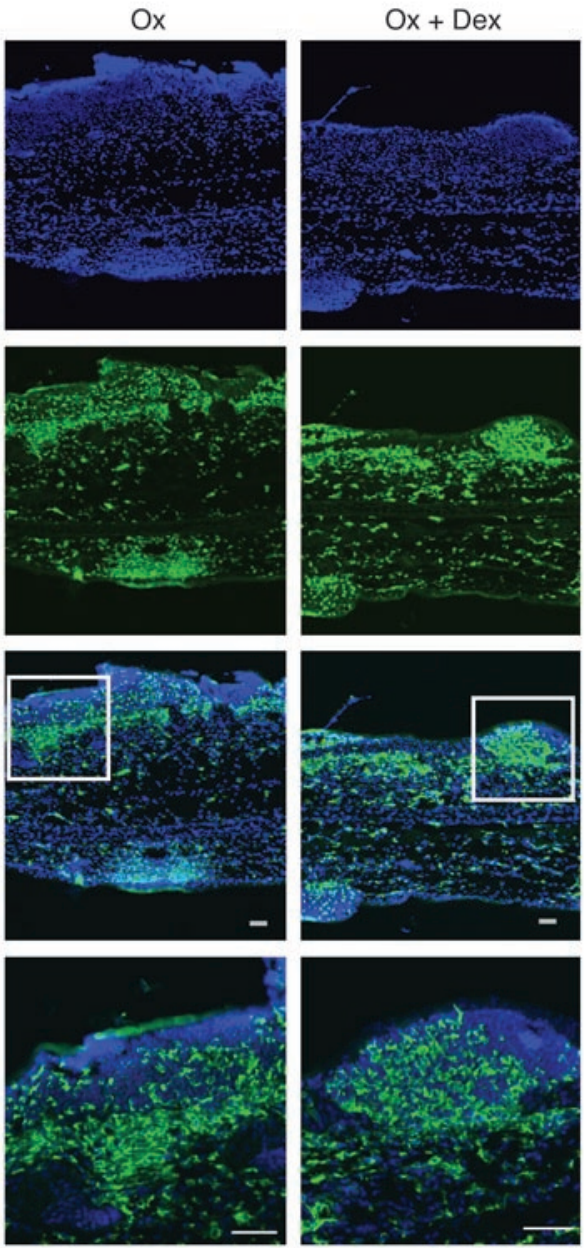

\section{Figure 5}

Reduction of macrophages by dexamethasone is impaired in the skin of GR dim mice. Confocal images of ear sections from wild-type and GR ${ }^{\text {dim }}$ mice sensitized with oxazolone followed by administration of oxazolone alone or together with dexamethasone and then stained with CD68 (green). Nuclei are stained in blue (DAPI). Bottom row shows magnification of boxed regions in the third row. Scale bars: $50 \mu \mathrm{m}$.

ated repression of CHS suggests that a functional DNA-binding competent GR in myeloid cells is required for this effect. Since the cellular composition of dexamethasone-treated wild-type and GR ${ }^{\mathrm{dim}}$ skin is different, it is not feasible to quantitatively determine the levels of inflammatory mediators per macrophage in tissue samples. Therefore, BMDMs from $\mathrm{GR}^{\mathrm{dim}}$ mice were studied ex vivo as a well defined macrophage model (40). Although these cells do not represent skin resident macrophages in every respect, these cells show the classic features of activated macrophages when stimulated with LPS, such as the expression of proinflammatory mediators (40) known to be involved in CHS (4). Our experiments revealed that suppression of several of these mediators by dexamethasone was impaired in $\mathrm{GR}^{\mathrm{dim}}$ macrophages. Moreover, when applied in vivo, these factors indeed partially counteracted dexamethasone-mediated repression of CHS. Collectively, using ex vivo-generated macrophages with a DNA binding-impaired GR, we identified proinflammatory genes, which are GC targets participating in the efficacy of steroid treatment of CHS. TNF- $\alpha$ release was effectively repressed by dexamethasone in $\mathrm{GR}^{\mathrm{dim}}$ macrophages, and admin- istration of recombinant TNF- $\alpha$ to the foot pads of mice did not significantly counteract GC-mediated suppression of CHS. Thus, TNF- $\alpha$ does not seem to be the important GC target, but it could still act in synergy with other chemokines or cytokines. In contrast, IL-1 $\beta$ release was not effectively repressed in $\mathrm{GR}^{\mathrm{dim}}$ macrophages. IL-1 $\beta$ is known to play a pivotal role in inflammatory responses (41), although the requirement for it in CHS depends on the specific hapten. While IL-1 $\beta$-knockout mice fail to mount a hypersensitivity response to low doses of trinitrochlorobenzene (42), they strongly respond to oxazolone (43). On the other hand, we found that exogenous application of IL-1 $\beta$ to oxazolone-treated mice slightly impaired the dexamethasone-mediated suppression. Studies with IL-1 $\beta$-knockout mice treated with oxazolone and dexamethasone could clarify this question. We also demonstrate that dexamethasone repression of MCP-1, a potent chemoattractant molecule, was significantly impaired in $\mathrm{GR}^{\mathrm{dim}}$ macrophages. While MCP-1-knockout animals are able to mount a normal CHS response, they fail to recruit leukocytes to the site of inflammation (44). This is in agreement with our finding that the number of macrophages 
WT
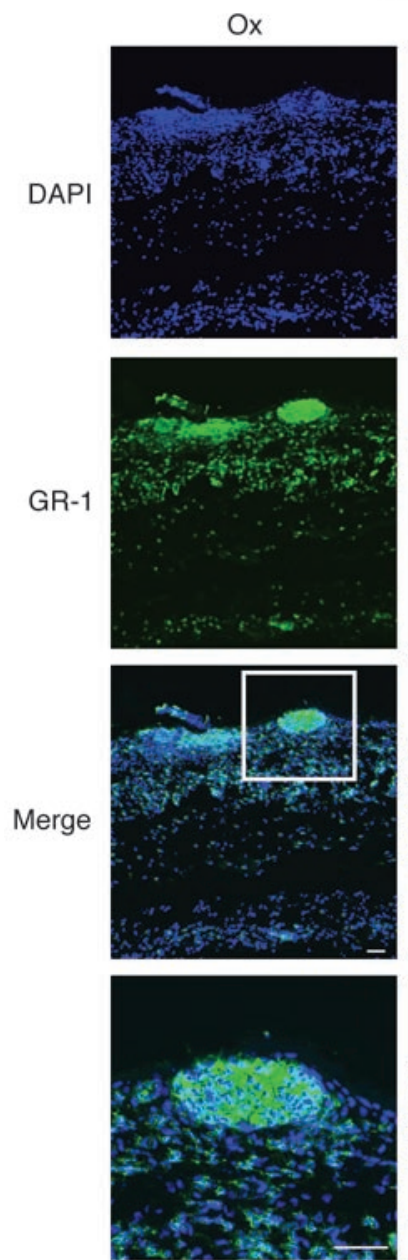

$\mathrm{Ox}+\mathrm{Dex}$
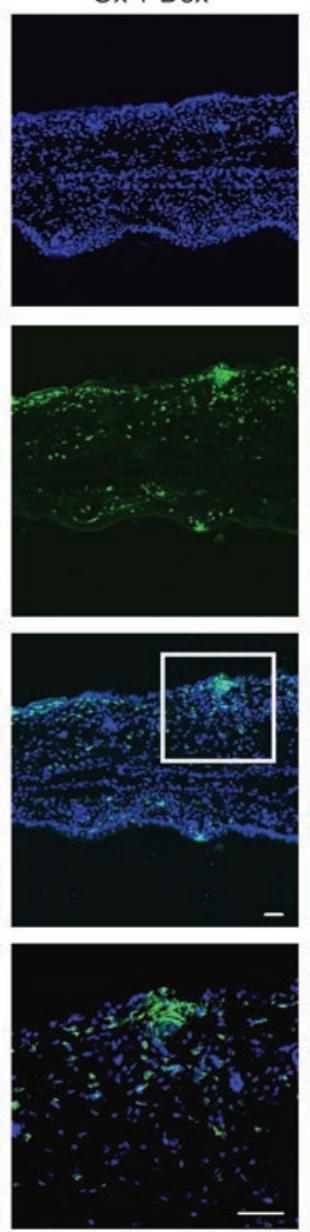

$\mathrm{GR}^{\mathrm{dim}}$
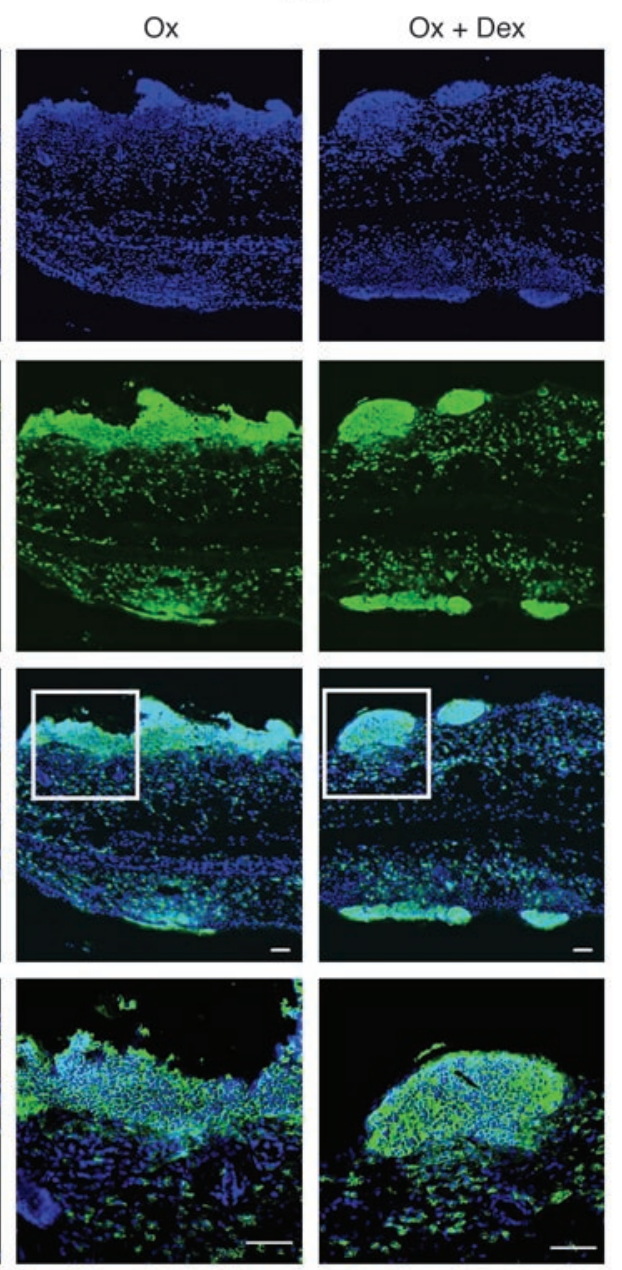

\section{Figure 6}

Reduction of neutrophil granulocytes by dexamethasone is hampered in the skin of GR dim mice. Confocal images of ears of the same specimens used in Figure 5, stained with antibodies against GR-1 (green). Nuclei are stained in blue (DAPI). Bottom row shows magnification of boxed regions in the third row. Scale bars: $50 \mu \mathrm{m}$.

in the inflamed ears of GR ${ }^{\text {dim }}$ mice was not reduced after dexamethasone treatment. The treatment with recombinant MCP-1 led to a partial relief of dexamethasone repression. Thus, inhibition of MCP-1 release is presumably relevant for the antiinflammatory activity of GCs. Downregulation of MIP-2 protein secretion by GCs was completely abolished in GR ${ }^{\text {dim }}$ BMDMs. MIP-2 is an attractant molecule for neutrophils, and neutralization of MIP-2 leads to an attenuation of virally induced hypersensitivity response (45). In addition, mice lacking the receptor for MIP-2 exhibit an impairment of CHS (46). Administration of recombinant MIP-2 to oxazolone- and dexamethasone-treated ears also causes a slight attenuation of dexamethasone-mediated suppression of CHS. Therefore, inhibition of MIP-2 could participate in diminishing the CHS response by GC via reducing infiltration of neutrophils. Similarly, IP-10 was hardly downregulated in GRdim cells by dexamethasone. IP-10 is also known to be required for a complete induction of CHS because the IP-10-knockout mice show a reduced swelling response $(13,47)$. IP-10 is induced during the challenge phase in keratinocytes and monocytic cells in the skin (48). Activated infiltrating T cells express CXCR3, the receptor for IP-10 (49), thus linking increased expression of IP-10 to the recruitment of sensitized $T$ cells to the site of inflammation. In line with our finding that GCs fail to repress IP-10 in GR dim cells, administration of recombinant IP-10 partially reversed the suppressive effect of dexamethasone on the CHS response. Thus, IP-10 could also be a crucial target for GC-mediated suppression of CHS.

In summary, several cytokines and chemokines participate in the impaired suppression of macrophage and/or neutrophil function in $\mathrm{GR}^{\mathrm{dim}}$ mice. Consistently, administration of each recombinant mediator to oxazolone-treated mice only partially reversed the suppressive effect of dexamethasone. Our data suggest that there is not a single target of the antiinflammatory action of GCs in CHS but that a network of inflammatory mediators is controlled by the GR dimer.

We have identified the critical cell types for GC action and determined that DNA binding by the GR is involved in the molecular mechanism that mediates the therapeutic effects of GCs in the treatment of allergic skin conditions. Our results indicate that the most promising strategy for the future will be to target genes 
A

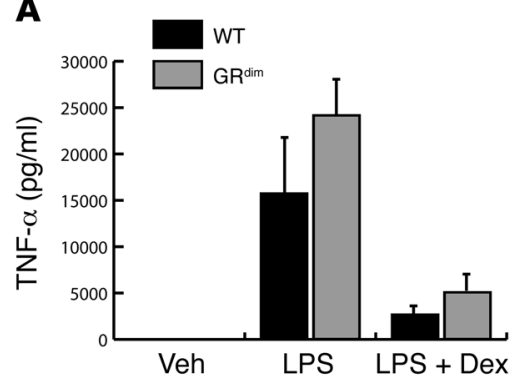

D

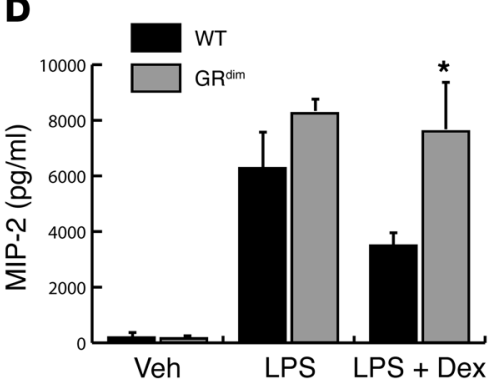

B

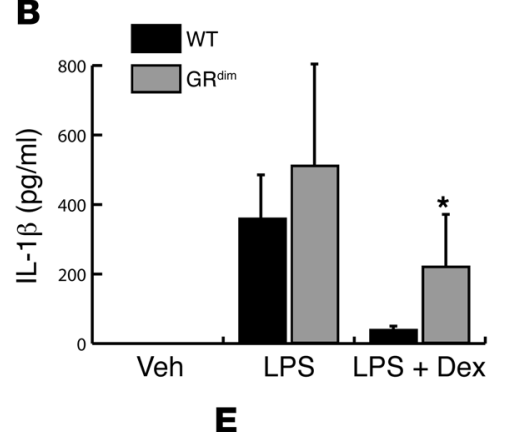

C

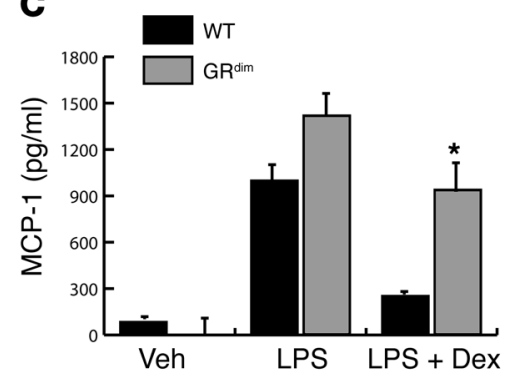

Figure 7

GC-mediated repression of cytokines and chemokines in LPS-activated BMDMs of wild-type and GRdim mice. BMDMs were treated with LPS or LPS and dexamethasone, and cytokine release in the supernatant was determined for TNF- $\alpha$ after 8 hours (A), for IL-1 $\beta$ after 8 hours (B), for MCP-1 after 24 hours (C), for MIP-2 after 8 hours (D), and for IP-10 after 24 hours (E). For IL-1 $\beta$, BMDMs were also treated with ATP in the last 30 minutes. Statistical analysis was performed for at least 3 independent experiments. ${ }^{*} P<0.05$.

in myeloid cells, in particular chemoattractant proteins and cytokines, which interfere with leukocyte migration to the site of hapten exposure. Interestingly, low molecular weight antagonists for chemokine receptors, such as CCR2-binding (50) MCP-1 or IL- $1 \beta$ receptor inhibitor (41), are being tested in animal models and in clinical trials and could serve as promising alternatives for steroid therapy in contact dermatitis.

\section{Methods}

Mice. For CHS experiments C57BL/6 and Balb/c mice were purchased from Charles River Laboratories. GR flox mice, which were described previously (22), were bred to the transgenic Cre lines lckCre (23), K14CreERT2 (21), and LysMCre (25). All lines were congenic to C57BL/6 for more than 10 generations. To quantify recombination, $\mathrm{GR}^{\mathrm{LysMCre}}$ mice were crossed to RA/EG mice and Cre-mediated recombination was determined by EGFP expression as described elsewhere (26). The generation of the GR dim mice was described previously (27). These mice were maintained in a mixed background of 129/Sv and C57BL/6.

All mice were maintained under standardized conditions with water and food ad libitum in a specific pathogen-free animal facility at the German Cancer Research Center (DKFZ) and the Leibniz Institute for Age Research, Fritz Lipmann Institute. The procedures for performing animal experiments were in accordance with the principles of the Arbeitsgemeinschaft der Tierschutzbeauftragten in Baden-Württemberg (ATBW; officials for animal welfare in Germany) and were approved by the Regierungspräsidium (Karlsruhe, Germany) and the Thüringer Landesamt für Lebensmittelsicherheit und Verbraucherschutz (TLLV Thüringen; Erfurt, Germany).

Reagents and antibodies. DNFB, FITC, and oxazolone were purchased from Sigma-Aldrich. Fluocinonide ointment (Topsym) was purchased from Grünenthal AG. Rat anti-mouse anti-CD4/PE, anti-CD8/APC, anti-CD11c/APC (NLDC-145), anti-GR-1/APC, anti-c-kit-APC, anti-
CD32/CD16 (2.4G2, Fc block), and isotype controls were obtained from BD Biosciences.

$P C R$. Bone marrow cell suspensions from $\mathrm{GR}^{\mathrm{LysMCre}}$;RA/EG mice were prepared as described in ref. 51. FACS analysis with anti-GR-1/APC antibody was performed according to standard procedures as described elsewhere (51). EGFP-positive and EGFP-negative GR-1+ cells were sorted with a FACSVantage cell sorter (BD Biosciences) and lysed as described in ref. 27. PCR from the lysates was performed at $63^{\circ} \mathrm{C}$ annealing with the primers GRflox-1 (forward) 5-GGCATGCACATTACTGGCCTTCT-3, GRflox-4 (reverse) 5-GTGTAGCAGCCAGCTTACAGGA-3, and GRflox-8 (reverse) 5-CCTTCTCATTCCATGTCAGCATGT-3. Subsequent gel electrophoresis allowed the detection of the null allele as a $390-\mathrm{bp}$ band, the GR ${ }^{\text {flox }}$ allele as a 275-bp band, and the wild-type allele as a 225-bp band.

Animal experimentation in CHS experiments. Induction of CHS was performed as described (5). After shaving, abdominal skin was painted with $25 \mu \mathrm{l}$ of $0.5 \%$ DNFB in acetone/olive oil (4:1) or $50 \mu \mathrm{l}$ of $5 \%$ oxazolone in acetone/olive oil (4:1). Five days later, the mice were challenged with $10 \mu \mathrm{l}$ of $0.2 \%$ DNFB or $1 \%$ oxazolone on the dorsal side of the left ear or on the left foot pad. Ear or foot thickness was measured before challenge and again after 24 hours with a thickness gauge (Mitutoyo), and the difference between the 2 readings was recorded as ear or foot swelling in $\mathrm{mm}$ or percentage of the thickness prior to treatment, depicted in Figures 1-4 and Figure 8 as "swelling in mm" or "\% of swelling". GC treatment was started after 5 days or at the time points indicated in Figures 1 and 2, starting with an i.p. injection of $25 \mu \mathrm{g}$ dexamethasone (Sigma-Aldrich) and subsequently continued with $20 \mathrm{mg} / \mathrm{l}$ dexamethasone in the drinking water to maintain hormone levels for 24 hours or the time period indicated in Figures 1 and 2. Alternatively, $5 \mu$ fluocinonide was topically applied 1 hour prior to hapten application. Recombinant TNF- $\alpha(0.1 \mu \mathrm{g})$, IL-1 $\beta(0.01 \mu \mathrm{g})$, MCP-1 $(0.1 \mu \mathrm{g}), \mathrm{MIP}-2(0.1 \mu \mathrm{g})$, or IP-10 (0.1 $\mu \mathrm{g})(\mathrm{R} \& \mathrm{D}$ Systems) was dissolved in PBS/0.1\% BSA and injected into the foot pad 1 hour prior to dexamethasone treatment. 
A

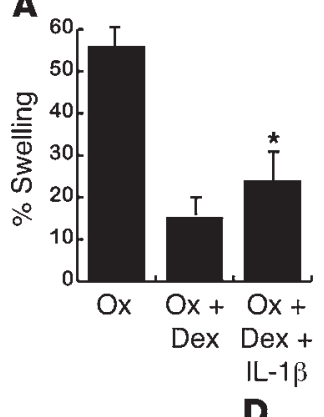

B

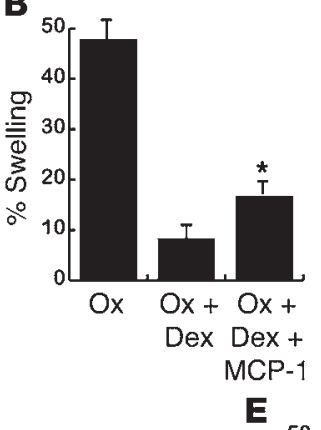

C

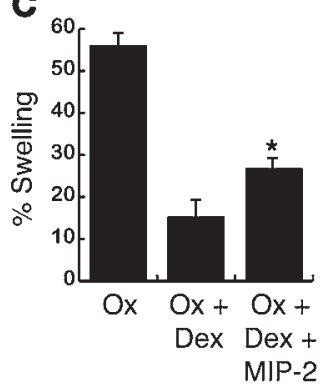

Figure 8

Cytokines and chemokines with impaired downregulation in GR ${ }^{\text {dim }}$ macrophages interfere with $\mathrm{GC}$-mediated suppression of $\mathrm{CHS}$. Mice were sensitized and challenged with oxazolone. During the challenge phase they were also treated either with dexamethasone (Ox+Dex) alone or with recombinant IL-1 $\beta$ (A), MCP-1 (B), MIP-2 (C), IP-10 (D), or TNF- $\alpha$ (E) at the concentration indicated in Methods. ${ }^{*} P<0.05(n=6)$.

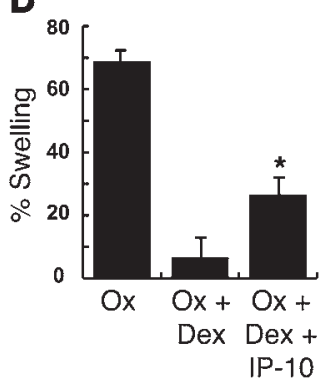

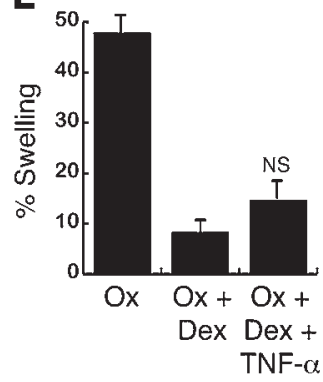

Assay for hapten-induced DC migration. Mice were treated as described above with dexamethasone and painted 24 hours later on the shaved back with $400 \mu \mathrm{l}$ of $0.5 \%$ FITC in acetone/dibutylphthalate (1:1; Sigma-Aldrich). Axillary, brachial, and inguinal lymph nodes were collected 18 hours later, and cell suspensions were prepared as previously described (52). Cell suspensions were subjected to staining with anti-CD11c/APC, anti-CD4/PE, and anti-CD8/PerCP. FACS analysis was performed using a FACSCalibur (BD Biosciences) and the data processed with FlowJo software (version 6.2; Tree Star Inc.).

Immunohistochemistry and histology. Confocal laser scanning microscopy was performed with $6-\mu \mathrm{m}$ cryostat sections of isolated ears 24 hours after oxazolone challenge that were embedded in Tissue-Tek (Sakura). Specimens were placed on glass slides and fixed with acetone for 10 minutes at $4{ }^{\circ} \mathrm{C}$. After rehydration in PBS supplemented with $2 \%$ FCS for 20 minutes at room temperature, specimens were incubated for 1 hour at room temperature with unlabeled primary antibodies against Gr-1 (BD Biosciences) or CD68 (AbD Serotec). Nuclei were counterstained with DAPI. Secondary antibodies (donkey anti-rat IgG-Alexa Fluor 488 [Invitrogen] and goat anti-hamster IgG-Cy3 [dianova $\mathrm{GmbH}$ ]) were applied for 30 minutes at room temperature. Sheets were mounted in Permafluor (Beckman Coulter) and viewed on a Zeiss Axiovert $200 \mathrm{M}$ microscope equipped with a confocal laser scanning head (LSM510). Pictures were taken and analyzed using LSM510 Image Examiner software (Zeiss AG).

Isolation and culture of BMDM, protein determination and quantitative RT-PCR analysis. BMDMs were obtained from 10-week-old wild-type and GR ${ }^{\text {dim }}$ mice. BM was cultured in DMEM (Invitrogen) supplemented with $10 \%$ FBS (Invitrogen) and 20\% L929 cell-conditioned medium as a source of M-CSF. The adherent cells were treated at day 10 with LPS $(100 \mathrm{ng} / \mathrm{ml})$ and dexamethasone $\left(10^{-6} \mathrm{M}\right)$ for the time points indicated in Figure 7 and Supplemental Figure 1. For determination of IL-1 $\beta$ release, cells were additionally treated with ATP (Sigma-Aldrich) 30 minutes before the time point indicated in Figure 7. Supernatant was collected and protein concentrations determined by ELISA for IP-10 (R\&D Systems), MIP-2 (R\&D Systems), IL-1 $\beta$ (BD Biosciences), and MCP-1 (BD Biosciences) or by cytometric bead array (Mouse Inflammation Kit; BD Biosciences) for TNF- $\alpha$ and IL- 6 according to the manufacturer's protocol.

For quantitative real time PCR analysis, cells were harvested and RNA extracted with RNeasy Mini Kit (QIAGEN) following the manufacturer's instructions. One $\mu \mathrm{g}$ of DNase I-treated RNA was used to generate cDNA by oligo(dT) priming. The following primers were used for quantitative PCR analysis: TNF- $\alpha, 5$-GCCTCTTCTCATTCCTGCTT-3 (forward) and 5-CTCCTCCACTTGGTGGTTTG-3 (reverse); IL-1 $\beta$, 5-GGGAAACAACAGTGGTCAGG-3 (forward) and 5-ATGTGCCATGGTTTCTTGTG-3 (reverse); MCP-1, 5-CCAGCTCTCTCTTCCTCCAC-3 (forward) and 5-CTGCTGCTGGTGATCCTCTT-3 (reverse); IP-10, 5-AGAGACATCCCGAGCCAAC-3 (forward) and 5-CTTCCCTATGGCCCTCATTC3 (reverse); MIP-2, 5-CTCAAGGGCGGTCAAAAAG-3 (forward) and 5-CGAGGCACATCAGGTACGA-3 (reverse); tubulin, 5-AATCGATGAGATCCGAAATG-3 (forward) and 5-TGCACTGATCAGACAGCTTG-3 (reverse). PCR was performed with an iCycler (Bio-Rad) using a SensiMix (dU) DNA kit containing SYBR green (Quantace). Quantification of results was performed with iQ5 Optical Systems Software version 1.0 (Bio-Rad) using the standard curve method. Relative expression levels were calculated in comparison with LPS-stimulated cells and depicted in Supplemental Figure 1 as a percentage of LPS-induced expression levels. Three to five independent experiments were performed and statistically analyzed using 2-tailed Student's $t$ test.

\section{Acknowledgments}

We would like to acknowledge Falk Weih and Helen Morrison for critical reading of the manuscript; Stefan Berger for designing the GR ${ }^{\text {flox }}$ PCR; Bernd Arnold for providing the RA/EG mice; Andrea Takacs, Katrin Buder, Susanne Ostermay, and Sabine Hübner for excellent technical assistance; and Sabine Matz for maintaining the mouse colonies. This work was supported by the Deutsche Forschungsgemeinschaft through Collaborative Research Centres SFB488, SFB405, and SFB636 and grants FOR Ot 165/2-2, GRK 791/1.02, GRK 484, Sachbeihilfe Tu-220/3, and Schu 51/7-2; by the Fonds der Chemischen Industrie; by the European Community through grant QLG1CT-2001-01574 and LSHM-CT-2005-018652 (CRESCENDO); by the Bundesministerium für Bildung und Forschung (BMBF) through Nationales Genomforschungsnetz (NGFN) grants FZK 01GS01117, 01GS0477, and KGCV1/01GS0416; by the GermanPolish cooperation project $01 \mathrm{GZ} 0310$ and $0313074 \mathrm{C}$ (systems 
biology); by the Nederlandse Organisatie voor Wetenschappelijk Onderzoek (NWO; grant VID 917.76.365); by the Landsteiner Foundation for Blood Transfusion Research (LSBR, 0414); and by the Austrian Fonds zur Förderung der wissenschaftlichen Forschung (grant SFB F28).

Received for publication January 24, 2006, and accepted in revised form February 20, 2007.

1. Leung, D.Y., Boguniewicz, M., Howell, M.D., Nomura, I., and Hamid, Q.A. 2004. New insights into atopic dermatitis. J. Clin. Invest. 113:651-657. doi:10.1172/JCI200421060.

2. Barnes, P.J. 1998. Anti-inflammatory actions of glucocorticoids: molecular mechanisms. Clin. Sci. (Lond.). 94:557-572.

3. Grabbe, S., and Schwarz, T. 1998. Immunoregulatory mechanisms involved in elicitation of allergic contact hypersensitivity. Immunol. Today. 19:37-44.

4. Wang, B., et al. 2003. Cytokine knockouts in contact hypersensitivity research. Cytokine Growth Factor Rev. 14:381-389.

5. Wang, B., et al. 2000. CD4+ Th1 and CD8+ type 1 cytotoxic $\mathrm{T}$ cells both play a crucial role in the full development of contact hypersensitivity. J. Immunol. 165:6783-6790.

6. Campos, R.A., et al. 2003. Cutaneous immunization rapidly activates liver invariant Valpha14 NKT cells stimulating B-1 B cells to initiate T cell recruitment for elicitation of contact sensitivity. J. Exp. Med. 198:1785-1796.

7. Campos, R.A., et al. 2006. Interleukin-4-dependent innate collaboration between iNKT cells and B-1 B cells controls adaptative contact sensitivity. Immunology. 117:536-547.

8. O’Leary, J.G., Goodarzi, M., Drayton, D.L., and von Andrian, U.H. 2006. T cell- and B cell-independent adaptive immunity mediated by natural killer cells. Nat. Immunol. 7:507-516.

9. Tsuji, R.F., et al. 2002. B cell-dependent T cell responses: IgM antibodies are required to elicit contact sensitivity. J. Exp. Med. 196:1277-1290.

10. Tsuji, R.F., et al. 2000. Early local generation of C5a initiates the elicitation of contact sensitivity by leading to early $\mathrm{T}$ cell recruitment. J. Immunol. 165:1588-1598.

11. McHale, J.F., Harari, O.A., Marshall, D., and Haskard, D.O. 1999. Vascular endothelial cell expression of ICAM- 1 and VCAM- 1 at the onset of eliciting contact hypersensitivity in mice: evidence for a dominant role of TNF-alpha. J. Immunol. 162:1648-1655.

12. Hwang, J.M., Yamanouchi, J., Santamaria, P., and Kubes, P. 2004. A critical temporal window for selectin-dependent CD4+ lymphocyte homing and initiation of late-phase inflammation in contact sensitivity. J. Exp. Med. 199:1223-1234.

13. Dufour, J.H., et al. 2002. IFN-gamma-inducible protein 10 (IP-10; CXCL10)-deficient mice reveal a role for IP-10 in effector T cell generation and trafficking. J. Immunol. 168:3195-3204.

14. Tuckermann, J.P., Kleiman, A., McPherson, K.G., and Reichardt, H.M. 2005. Molecular mechanisms of glucocorticoids in the control of inflammation and lymphocyte apoptosis. Crit. Rev. Clin. Lab. Sci. 42:71-104.

15. Beato, M., Herrlich, P., and Schutz, G. 1995. Steroid hormone receptors: many actors in search of a plot. Cell. 83:851-857.

16. Herrlich, P. 2001. Cross-talk between glucocorticoid receptor and AP-1. Oncogene. 20:2465-2475.

17. Reichardt, H.M., et al. 2001. Repression of inflammatory responses in the absence of DNA binding by the glucocorticoid receptor. $Е M B O J$. 20:7168-7173.

18. Tuckermann, J.P., et al. 1999. The DNA binding-
Address correspondence to: Günther Schütz, German Cancer Research Center, Division of Molecular Biology of the Cell I, Im Neuenheimer Feld 280, D-69120 Heidelberg, Germany. Phone: 49-6221-423411; Fax: 49-6221-423470; E-mail: g.schuetz@dkfz.de. Or to: Jan P. Tuckermann, Leibniz Institute for Age Research, Fritz-Lipmann-Institute, Division of Tissue-Specific Hormone Action, Beutenberg Str. 11, D-07745 Jena, Germany. Phone: 49-3641-656134; Fax: 49-3641-656133; E-mail: jan@fli-leibniz.de. independent function of the glucocorticoid receptor mediates repression of AP-1-dependent genes in skin. J. Cell Biol. 147:1365-1370.

19. Cumberbatch, M., Dearman, R.J., and Kimber,I. 1999. Inhibition by dexamethasone of Langerhans cell migration: influence of epidermal cytokine signals. Immunopharmacology. 41:235-243.

20. Askenase, P.W. 2001. Yes T cells, but three different $T$ cells (alphabeta, gammadelta and NK T cells), and also B-1 cells mediate contact sensitivity. Clin. Exp. Immunol. 125:345-350.

21. Indra, A.K., et al. 2000. Targeted somatic mutagenesis in mouse epidermis. Horm. Res. 54:296-300.

22. Tronche, F., et al. 1999. Disruption of the glucocorticoid receptor gene in the nervous system results in reduced anxiety. Nat. Genet. 23:99-103.

23. Gu, H., Marth, J.D., Orban, P.C., Mossmann, H., and Rajewsky, K. 1994. Deletion of a DNA polymerase beta gene segment in T cells using cell typespecific gene targeting. Science. 265:103-106.

24. Baumann, S., et al. 2005. Glucocorticoids inhibit activation-induced cell death (AICD) via direct DNA-dependent repression of the CD95 ligand gene by a glucocorticoid receptor dimer. Blood. 106:617-625

25. Clausen, B.E., Burkhardt, C., Reith, W., Renkawitz, R., and Forster, I. 1999. Conditional gene targeting in macrophages and granulocytes using LysMcre mice. Transgenic Res. 8:265-277.

26. Constien, R., et al. 2001. Characterization of a novel EGFP reporter mouse to monitor Cre recombination as demonstrated by a Tie2 Cre mouse line. Genesis. 30:36-44.

27. Reichardt, H.M., et al. 1998. DNA binding of the glucocorticoid receptor is not essential for survival. Cell. 93:531-541.

28. Macatonia, S.E., Knight, S.C., Edwards, A.J., Griffiths, S., and Fryer, P. 1987. Localization of antigen on lymph node dendritic cells after exposure to the contact sensitizer fluorescein isothiocyanate. Functional and morphological studies. J. Exp. Med. 166:1654-1667.

29. Hauser, C. 1990. Cultured epidermal Langerhans cells activate effector $\mathrm{T}$ cells for contact sensitivity. J. Invest. Dermatol. 95:436-440.

30. Biedermann, T., et al. 2000. Mast cells control neutrophil recruitment during $T$ cell-mediated delayed-type hypersensitivity reactions through tumor necrosis factor and macrophage inflammatory protein 2. J. Exp. Med. 192:1441-1452.

31. Pasparakis, M., et al. 2002. TNF-mediated inflammatory skin disease in mice with epidermis-specific deletion of IKK2. Nature. 417:861-866.

32. Albanesi, C., Scarponi, C., Giustizieri, M.L., and Girolomoni, G. 2005. Keratinocytes in inflammatory skin diseases. Curr. Drug Targets Inflamm. Allergy. 4:329-334.

33. Piguet, P.F., Grau, G.E., Hauser, C., and Vassalli, P. 1991. Tumor necrosis factor is a critical mediator in hapten induced irritant and contact hypersensitivity reactions. J. Exp. Med. 173:673-679.

34. Enk, A.H., and Katz, S.I. 1992. Early molecular events in the induction phase of contact sensitivity. Proc. Natl. Acad. Sci. U. S. A. 89:1398-1402.

35. Grabbe, S., Steinbrink, K., Steinert, M., Luger, T.A., and Schwarz, T. 1995. Removal of the majority of epidermal Langerhans cells by topical or systemic steroid application enhances the effector phase of murine contact hypersensitivity. J. Immunol. 155:4207-4217.

36. Kaplan, D.H., Jenison, M.C., Saeland, S., Shlomchik, W.D., and Shlomchik, M.J. 2005. Epidermal langerhans cell-deficient mice develop enhanced contact hypersensitivity. Immunity. 23:611-620.

37. Stratis, A., et al. 2006. Pathogenic role for skin macrophages in a mouse model of keratinocyteinduced psoriasis-like skin inflammation. J. Clin. Invest. 116:2094-2104. doi:10.1172/JCI27179.

38. Wang, H., et al. 2006. Activated macrophages are essential in a murine model for $\mathrm{T}$ cell-mediated chronic psoriasiform skin inflammation. J. Clin. Invest. 116:2105-2114. doi:10.1172/JCI27180.

39. Bullard, D.C., et al. 1996. A polygenic mouse model of psoriasiform skin disease in CD18-deficient mice. Proc. Natl. Acad. Sci. U. S. A. 93:2116-2121.

40. Lang, R., Patel, D., Morris, J.J., Rutschman, R.L., and Murray, P.J. 2002. Shaping gene expression in activated and resting primary macrophages by IL- 10 . J. Immunol. 169:2253-2263.

41. Dinarello, C.A. 1996. Biologic basis for interleukin-1 in disease. Blood. 87:2095-2147.

42. Shornick, L.P., et al. 1996. Mice deficient in IL-1beta manifest impaired contact hypersensitivity to trinitrochlorobenzone. J. Exp. Med. 183:1427-1436.

43. Zheng, H., et al. 1995. Resistance to fever induction and impaired acute-phase response in interleukin-1 beta-deficient mice. Immunity. 3:9-19.

44. Lu, B., et al. 1998. Abnormalities in monocyte recruitment and cytokine expression in monocyte chemoattractant protein 1-deficient mice. J. Exp. Med. 187:601-608.

45. Tumpey, T.M., Fenton, R., Molesworth-Kenyon, S., Oakes, J.E., and Lausch, R.N. 2002. Role for macrophage inflammatory protein 2 (MIP-2), MIP1alpha, and interleukin-1alpha in the delayed-type hypersensitivity response to viral antigen. J. Virol. 76:8050-8057.

46. Cattani, F., et al. 2006. The role of CXCR2 activity in the contact hypersensitivity response in mice. Eur. Cytokine Netw. 17:42-48.

47. Dilulio, N.A., et al. 1999. Groalpha-mediated recruitment of neutrophils is required for elicitation of contact hypersensitivity. Eur. J. Immunol. 29:3485-3495.

48. Mitsui, G., et al. 2003. Kinetic profiles of sequential gene expressions for chemokines in mice with contact hypersensitivity. Immunol. Lett. 86:191-197.

49. Qin, S., et al. 1998. The chemokine receptors CXCR3 and CCR5 mark subsets of T cells associated with certain inflammatory reactions. J. Clin. Invest. 101:746-754.

50. Brodmerkel, C.M., et al. 2005. Discovery and pharmacological characterization of a novel rodentactive CCR2 antagonist, INCB3344. J. Immunol. 175:5370-5378.

51. Bauer, A., et al. 1999. The glucocorticoid receptor is required for stress erythropoiesis. Genes Dev. 13:2996-3002.

52. Robbiani, D.F., et al. 2000. The leukotriene C(4) transporter MRP1 regulates CCL19 (MIP-3beta, ELC)-dependent mobilization of dendritic cells to lymph nodes. Cell. 103:757-768. 